\title{
Decursin from Angelica gigas Nakai Blocks hKv1.5 Channel
}

\author{
Yong Geun Kwak', Bok-Hee Choi ${ }^{2}$, Dae Keun Kim¹ and Jae Soon Eun ${ }^{1, *}$ \\ ${ }^{1}$ College of Pharmacy, Woosuk University, Samrye 565-701, \\ ${ }^{2}$ Department of Pharmacology, Chonbuk National University Medical School, Institute for Medical Research, Cheonju 561-756, \\ Republic of Korea
}

\begin{abstract}
Decursin was purified from Angelica gigas Nakai, and its effects on the human Kv1.5 (hKv1.5) currents were recorded in mouse fibroblasts (Ltk ${ }^{-}$cells) by whole-cell patch-clamp technique. Decursin inhibited hKv1.5 current in a concentration-dependent manner, with an $\mathrm{IC}_{50}$ value of $2.7 \mu \mathrm{M}$ at $+60 \mathrm{mV}$. Decursin accelerated the inactivation kinetics of the hKv1.5 channel, and slowed the deactivation kinetics of the hKv1.5 current, resulting in a tail crossover phenomenon. Also, decursin inhibited the hKv1.5 current in a use-dependent manner. These results strongly suggest that decursin is a kind of open-channel blocker of the hKv1.5 channel.
\end{abstract}

Key Words: hKv1.5 channel blocker, Decursin, Angelica gigas Nakai

\section{INTRODUCTION}

Atrial fibrillation is the most frequent cardiac arrhythmia that can result in serious morbidity (Chung et al., 2001). It is well known that various $\mathrm{K}^{+}$channels regulate the action potential duration and $\mathrm{K}^{+}$channel genes are differentially expressed depending on the regions of the heart. The main $\mathrm{Kv}$ channel genes expressed in the human heart are the hKv1.4, hKv1.5, hKv4.3 and HERG genes. All these genes are highly expressed in both the atrium and ventricle, whereas the hKv1.5 gene is preferentially expressed in the human atrium. Furthermore, the electrophysiological and pharmacological characteristics of the current generated by hKv1.5 channels is similar to the ultra-rapid delayed rectifier $\mathrm{K}^{+}$current $\left(\mathrm{I}_{\mathrm{KUR}}\right)$ recorded in human atrial myocytes (Fedida et al., 1998). Thus, the hKv1.5 channel is thought to be an unique target for atrial fibrillation. In the present study, we tested decursin from the roots of $A n-$ gelica gigas Nakai (Umbelliferae) on $\mathrm{K}^{+}$currents expressed in Ltk $^{-}$cells. $A$. gigas Nakai has been used as a traditional medicine for treatment of anemia, a sedative and tonic agent (Yook, 1990). Earlier investigations on the chemical constituents of $A$. gigas mainly dealt with the isolation of coumarins (Ryu et al., 1990; Jung et al., 1991; Pachaly et al., 1996; Lee et al., 2002). The literature survey revealed that several pharmacological works, anti-tumor, inhibition of hepatic microsomal drug metabolizing enzyme, and inhibition of acetylcholinesterase activities, have been carried out on $A$. gigas (Shin et al., 1996; Kang et al., 2001; Lee et al., 2003; Lee et al., 2009;
Kim et al., 2010). Our studies have focused on the development of antiarrhythmic drug, and we previously reported that papaverine (Choe et al., 2003), oxypeucedanin (Eun et al., 2005b), psoralen and their derivatives (Eun at al., 2005a; Eun et al., 2007) and torilin (Kwak et al., 2006) inhibited the hKv1.5 current. The present study was examined to investigate the effect of decursin from $A$. gigas on hKv1.5 channels using the whole-cell patch-clamp technique.

\section{MATERIALS AND METHODS}

\section{General procedure}

All the ${ }^{1} \mathrm{H}$ - and ${ }^{13} \mathrm{C}$-NMR spectra were determined on a JEOL JMN-EX 400 spectrometer. The EI/MS (70eV) spectrum was determined on a VG-VSEQ mass spectrometer (VG Analytical, UK). The TLC was carried out on precoated silica gel $\mathrm{F}_{254}$ plates (Merck, Darmstadt, Germany), and the silica gel for column chromatography was Kiesel gel 60 (230-400 mesh, Merck). The column used for LPLC was the Lobar A (Merck Lichroprep Si $60,240-10 \mathrm{~mm}$ ). All the other chemicals and solvents were of analytical grade and they were used without further purification.

\section{Plant materials}

The roots of $A$. gigas were purchased from Namchangdang, Jeonju, Korea. A voucher specimen is deposited in the herbarium of college of pharmacy, Woosuk University (WSU-

\section{www.biomolther.org}

Open Access DOI: 10.4062/biomolther.2011.19.1.033

pISSN: 1976-9148 elSSN: 2005-4483

Copyright $\odot 2011$ The Korean Society of Applied Pharmacology
Received Oct 14, 2010 Revised Dec 20, 2010 Accepted Dec 22, 2010

*Corresponding Author
E-mail: jseun@mail.woosuk.ac.kr
Tel: +82-63-290-1569, Fax: +82-63-290-1812 
04-034).

\section{Extraction and isolation}

The air-dried plant materials (300 g) were ground and extracted with $\mathrm{MeOH}$ under $50^{\circ} \mathrm{C}$. The resultant $\mathrm{MeOH}$ extract $(52 \mathrm{~g})$ followed by the successive solvent partition to give methylene chloride $(20 \mathrm{~g}), n$-BuOH $(18 \mathrm{~g})$ and $\mathrm{H}_{2} \mathrm{O}$ soluble fractions. Methylene chloride soluble fraction showed the most significant hKv1.5 current inhibitory activity. This fraction was chromatographed over silica gel column using a solvent system $n$-hexnae- $\mathrm{CH}_{2} \mathrm{Cl}_{2}$-EtOAc $(3: 2: 1)$ as an eluent to give three subfractions, Subfraction 2 was purified by JAI-ODS column $(\mathrm{MeOH})$ to give compound 1 (150 mg).

\section{Compound 1 (Decursin, Fig. 1)}

Colorless needles $(\mathrm{MeOH}) ; 95^{\circ} \mathrm{C}$, the ${ }^{1} \mathrm{H}-\mathrm{NMR}$ and ${ }^{13} \mathrm{C}$ NMR data we obtained were in good agreement with the literature values (Lee et al., 2003).<smiles>CC(C)=CC(=O)O[C@H]1Cc2cc3ccc(=O)oc3cc2OC1(C)C</smiles>

Fig. 1. Chemical structure of Decursin.

\section{A. Control}

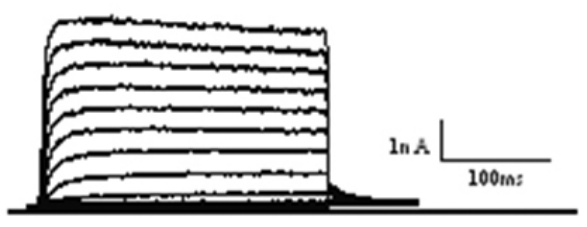

\section{B. Decursin $10 \mu M$}

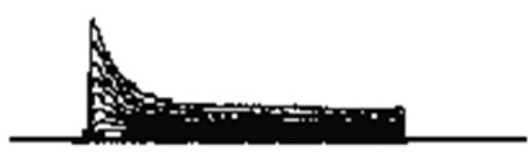

C.

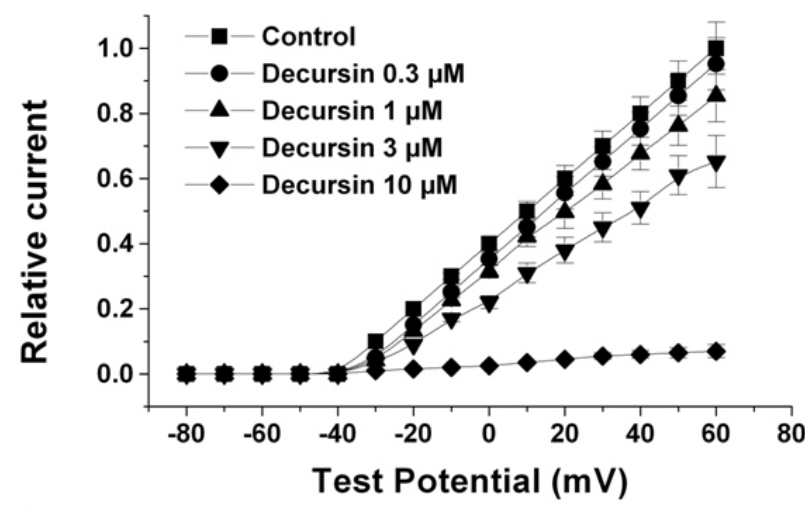

D.

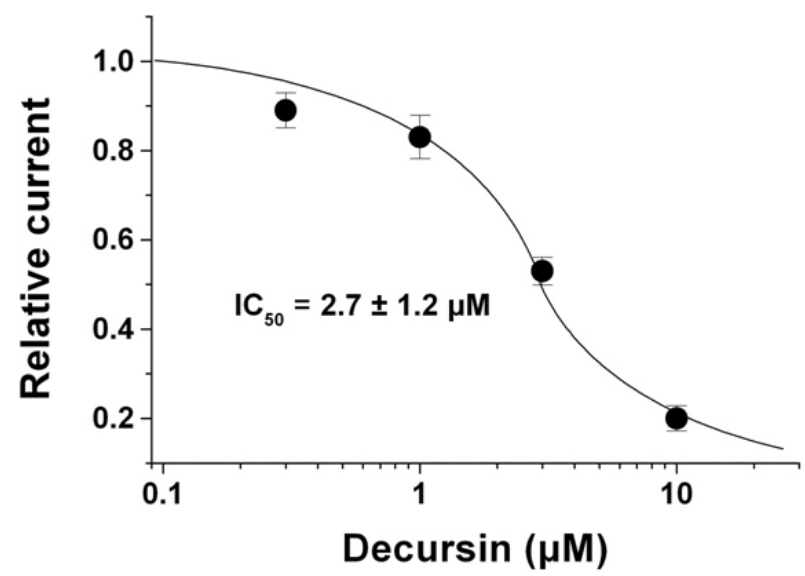

Fig. 2. Effects of decursin on the hKv1.5 current expressed in Ltk $^{-}$ cell line. hKv1.5 current traces were recorded before $(A)$ and 20 min after exposure to $1 \mu \mathrm{M}$ decursin (B). (C) The resultant I-V relationship of the steady-state current taken at the end of the depolarizing pulses. (D) Concentration-response relationship of hKv1.5 block by decursin. Each point with a vertical bar denotes the mean \pm S.E.M. 


\section{Cell culture and transfection}

The method is the same as that described previously (Snyders et al., 1993). The transfected cells were cultured in Dulbecco's modified Eagle's medium supplemented with $10 \%$ horse serum and $0.25 \mathrm{mg} / \mathrm{ml}$ of $\mathrm{G} 418$, under a $5 \% \mathrm{CO}_{2}$ atmosphere. Before the experimentals, the subconfluent cultures were incubated with $2 \mu \mathrm{M}$ dexamethasone for $12 \mathrm{~h}$ to induce expression of hKv1.5 channels.

\section{Electrical recording}

hKv1.5 currents in Ltk $^{-}$cells were recorded at room temperature $\left(20-22^{\circ} \mathrm{C}\right)$ using the whole-cell configuration of the patch clamp technique (Kwak et al., 1999) with an Axopatch200B patch clamp amplifier (Axon Ins, Foster City, CA, USA). Currents were sampled at 1 to $10 \mathrm{kHz}$ after an anti-alias filtering was done at 0.5 to $5 \mathrm{kHz}$. Data acquisition and command potentials were controlled by pClamp 6.0.3 software (Axon Ins). Junction potentials were zeroed with the electrode in the standard bath solution. Gigaohm seal formation was achieved by suction and, after establishing the whole cell configuration, the capacitive transients were elicited by a symmetrical $10 \mathrm{mV}$ voltage clamp in steps from $-80 \mathrm{mV}$, and they were recorded at $50 \mathrm{kHz}$ for the calculation of cell capacitance. Whole cell currents of -1 to $4 \mathrm{nA}$ and series resistances of 2 to $3 \mathrm{M} \Omega$ were used for the analysis. The intracellular pipette-filling solution for whole cell mode contained $100 \mathrm{mM} \mathrm{KCl}, 10 \mathrm{mM}$ HEPES, $5 \mathrm{mM} \mathrm{K}_{4}$ BAPTA, $5 \mathrm{mM} \mathrm{K}_{2}$ ATP and $1 \mathrm{mM} \mathrm{MgCl}_{2}(\mathrm{pH}$ 7.2). The extracellular solution contained $130 \mathrm{mM} \mathrm{NaCl}, 4 \mathrm{mM}$ $\mathrm{KCl}, 1.8 \mathrm{mM} \mathrm{CaCl}_{2}, 1 \mathrm{mM} \mathrm{MgCl}, 10 \mathrm{mM}$ HEPES and $10 \mathrm{mM}$ glucose $(\mathrm{pH} 7.35)$. The current traces were recorded with a depolarizing pulse of $+50 \mathrm{mV}$ from a holding potential of -80 $\mathrm{mV}$, followed by a repolarizing pulse of $-50 \mathrm{mV}$ in the Ltk cells. To observe the concentration-dependent block of hKv1.5 channel currents by decursin, steady-state currents taken at the end of the depolarizing pulse of $+50 \mathrm{mV}$ were normalized to the control obtained in the absence of decursin.

\section{Statistical analysis}

All of the data are presented as mean \pm S.E.M. The Student's $t$-test and analysis of variance (ANOVA) were used to calculate the statistical significant. Differences were considered to be statistically significant if a $p$-value of $<0.05$ was obtained.

\section{RESULTS AND DISCUSSION}

A selective block of the hKv1.5 current results in a significant prolongation of the action potential (Wang et al., 1994). Fig. 2 demonstrates a representative experiment to examine the effects of decursin on the hKv1.5 channel currents expressed in the $\mathrm{Ltk}^{-}$cells. The membrane potentials were held at $-80 \mathrm{mV}$ and $250-\mathrm{ms}$ depolarizing pulses from -80 to $+60 \mathrm{mV}$ in $10 \mathrm{mV}$ steps were applied every $20 \mathrm{~s}$. The outward currents were followed by decaying outward tail currents upon repolarization to $-50 \mathrm{mV}$. In control conditions, a depolarization positive to -40 $\mathrm{mV}$ elicited outward. At $+60 \mathrm{mV}$, after the current reached the maximum, it declined slowly during the maintained depolarization (Fig. 2A). The peak current amplitude was affected much less than the steady-state current amplitude. The inhibition of hKv1.5 appeared within $20 \mathrm{~s}$ of the application of the drug and reached a new steady state within $2 \mathrm{~min}$. The washout of de- cursin was obtained within $4 \mathrm{~min}$, and the currents recovered to $92.1 \pm 4.7 \%(n=8)$ of the control value. Fig. $2 \mathrm{C}$ shows the steady-state I-V relationship for the hKv1.5 channels under control conditions and in the presence of decursin $(10 \mu \mathrm{M})$, and the I-V relations were constructed by plotting the current amplitudes measured at the end of 250-ms depolarizations as a function of the test pulse voltages. The hKv1.5 current was blocked by decursin over the whole potential range for activation. Thus, decursin significantly shifted the voltage dependence of channel opening to more hyperpolarized potentials, as reported for the action of two other drugs (Perchenet et al., 2000; Choe, et al., 2003). The block of hKv1.5 by decursin was in a concentration-dependent manner, as shown in Fig. 2D. Steady-state currents were measured at the end of depolarizing pulse of $+60 \mathrm{mV}$ to construct the concentration-response curve. Plots of steady-state current as a function of decursin concentration were fitted to the Hill equation. For decursininduced block, a half-maximal inhibitory concentration $\left(\mathrm{IC}_{50}\right)$ and Hill coefficient were $2.7 \pm 1.2 \mu \mathrm{M}$ and 1.31 , respectively $(n=8)$ (Fig. 2D). Drugs that block ion channels often alter the voltage dependence. Voltage-dependence of the drugs acting on ion channels is sometimes very useful in evaluating the clinical applications of the drugs. The voltage dependence of activation (Fig. 3A) and inactivation (Fig. 3B) were analyzed in the absence or presence of decursin $(3 \mu \mathrm{M})$. The activation
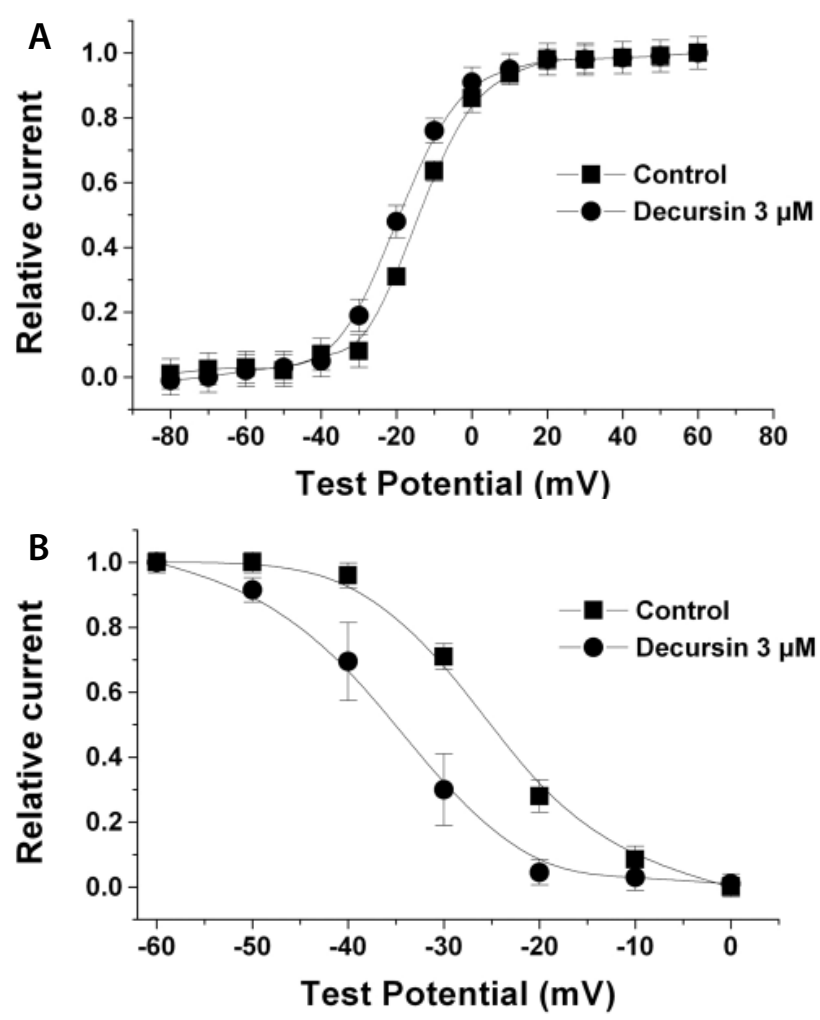

Fig. 3. Effects of decursin on the activation and inactivation of the hKv1.5 current. The activation curve (A) was obtained from the deactivating tail current amplitudes at $-50 \mathrm{mV}$, following 250 $\mathrm{ms}$ depolarizing steps to potentials between -80 to $+60 \mathrm{mV}$. The inactivation curve $(B)$ was obtained from the depolarizing steps to potentials between $-60 \mathrm{mV}$ to $0 \mathrm{mV}$, in steps of $10 \mathrm{mV}$. Each point with a vertical bar denotes the mean \pm S.E.M 
curve was unchanged in the presence of decursin: The values of $V_{1 / 2}$ for the activation were $-13.7 \pm 1.5$ and $-17.5 \pm 1.8 \mathrm{mV}$ $(n=8)$, without and with decursin, respectively. The values of $k$ were not significantly different $7.6 \pm 0.9 \mathrm{mV}$ for control and $9.1 \pm 1.2 \mathrm{mV}$ for decursin $(n=8)$. Under control conditions, $V_{1 / 2}$ and $k$ of the steady-state inactivation curve measured -24.3 $\pm 1.3 \mathrm{mV}$ and $4.8 \pm 0.9 \mathrm{mV}(\mathrm{n}=8)$, respectively. After the addition of decursin, the $V_{1 / 2}$ and $k$ of the steady-state inactivation curve changed to $-37.1 \pm 2.1 \mathrm{mV}$ and $4.3 \pm 1.2 \mathrm{mV}(\mathrm{n}=8)$, respectively.

To quantify the voltage dependence of the decursin-induced block of hKv1.5, the relative current $I_{\text {cytochalasin } B} / I_{\text {control }}$ was plotted as a function of membrane potential $(n=5)$ (Fig. 4). In the presence of decursin, the blockade increased steeply between -30 and $0 \mathrm{mV}$, which corresponds to the voltage range of the channel opening (Snyders et al., 1993). However, there was no additional inhibition of hKv1.5 by decursin in the range of voltages between 0 and $+60 \mathrm{mV}$, a range where the channels are fully activated. These data suggest that decursin binds primarily to the open state of the hKv1.5 channel. We tested the channel state-dependency of hKv1.5 block by decursin (Fig. 5). Fig. 5 shows the superposition of the tail currents obtained

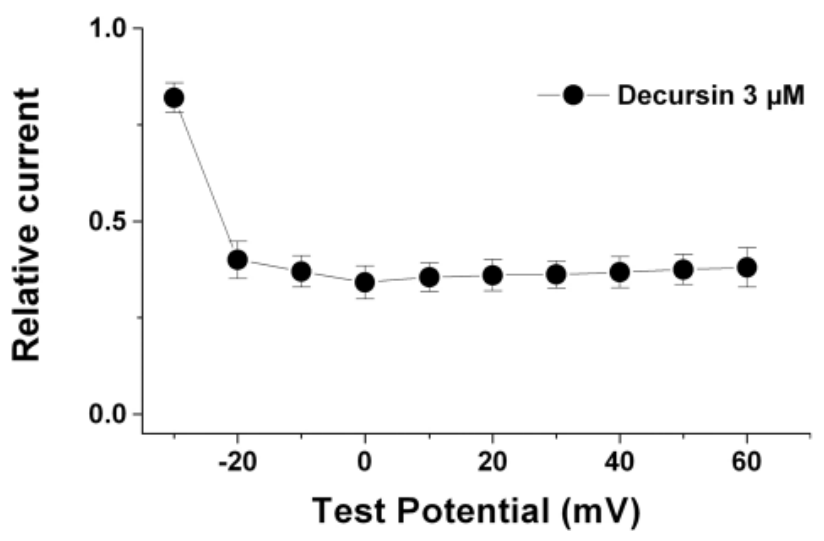

Fig. 4. Voltage-dependent block of hKv1.5 expressed in $\mathrm{Ltk}^{-}$cells by decursin. The voltage protocol consisted of $250-\mathrm{ms}$ depolarizing pulses from -20 to $+60 \mathrm{mV}$, in $10-\mathrm{mV}$ increments, from a holding potential of $-80 \mathrm{mV}$, with repolarizing to $-50 \mathrm{mV}$ for $400 \mathrm{~ms}$. Relative currents were obtained from the $I_{\text {decursin }} / I_{\text {control }}$ ratio at each depolarizing potential in the absence and presence of decursin. Each point with a vertical bar denotes the mean \pm S.E.M.

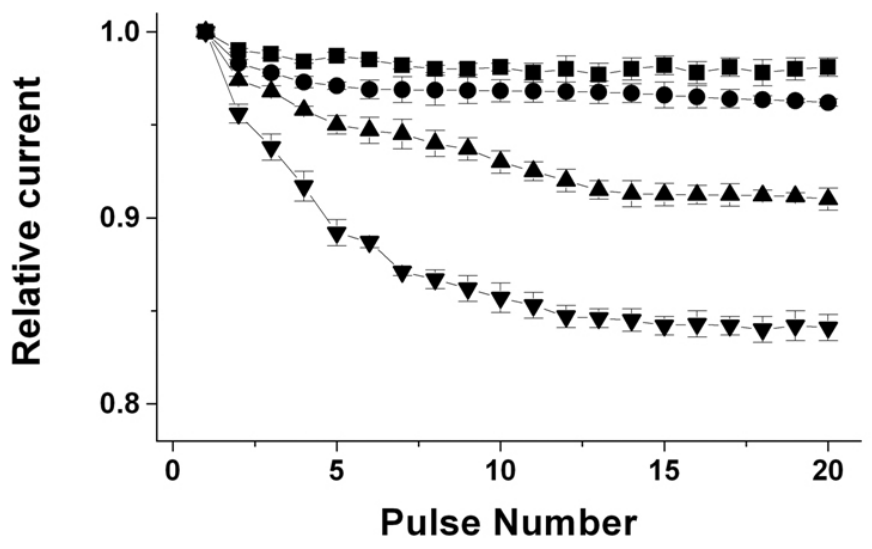

with a depolarizing pulse of $+50 \mathrm{mV}$ from a holding potential of $-80 \mathrm{mV}$, followed by a repolarizing pulse of $-50 \mathrm{mV}$ under control conditions and in the presence of decursin. In the presence of decursin, the initial tail current was decreased, with the subsequent slower decline resulting in a "crossover" phenomenon with the control tracing. This suggests that decursin acts as an open channel blocker on the hKv1.5 channel. On the basis of all these results, we suggest that decursin blocks hKv1.5 currents not by its known-mechanism, but rather via a direct one-to-one interaction between the drug and the channel in the open state, like as papaverine (Choe et al., 2003), oxypeucedanin (Eun et al., 2005b), psoralen (Eun et al., 2005a; Eun et al., 2007) and torilin (Kwak et al., 2006). The use-dependence of decursin-induced inhibition of the hKv1.5 channel was investigated. The current traces were produced by 20 repetitive applications of depolarizing pulses at two different frequencies, 1 and $2 \mathrm{~Hz}$. As shown in Fig. 6, the peak amplitude of the hKv1.5 current decreased slightly at a frequency of 1 and $2 \mathrm{~Hz}$ under control conditions. In the presence of decur$\sin (3 \mu \mathrm{M})$, the peak amplitude of the hKv1.5 current progressively decreased at 1 and $2 \mathrm{~Hz} \mathrm{~Hz}(n=4)$ respectively. Thus, the degree of inhibition of decursin on hKv1.5 increased at

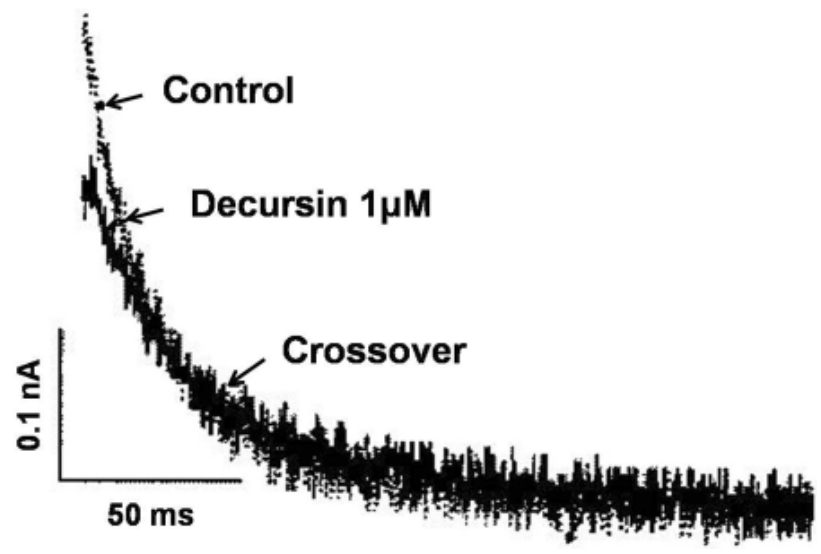

Fig. 5. Effects of decursin on the deactivation kinetics of hKv1.5 current expressed in Ltk ${ }^{-}$cells. Deactivation kinetics were investigated during a repolarizing step of $-50 \mathrm{mV}$ for $400 \mathrm{~ms}$, after a 250 $\mathrm{ms}$ depolarizing step to $+60 \mathrm{mV}$. From a holding potential of -80 $\mathrm{mV}$. By superimposing the tail currents in the absence and presence of decursin, a tail crossover phenomenon (indicated by the arrow) was observed.

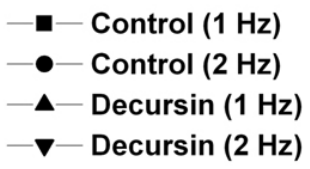

Fig. 6. Use-dependent inhibition of hKv1.5 expressed in Ltk $^{-}$cells by decursin. The plot of the normalized peak amplitudes of the currents, under control conditions and in the presence of decursin $(3 \mu \mathrm{M})$, at every pulse versus the pulse numbers in the pulse train. 
the pulse frequency increased, showing that decursin blocks hKv1.5 channels in a frequency-dependent manner.

The main $\mathrm{Kv}$ channel genes expressed in the human heart are the hKv1.4, hKv1.5, hKv4.3 and HERG genes. All these genes are highly expressed in both the atrium and ventricle, whereas the hKv1.5 gene is preferentially expressed in the human atrium. Furthermore, the electrophysiological and pharmacological characteristics of the current generated by $\mathrm{hKv} 1.5$ channels is similar to the $\mathrm{I}_{\mathrm{KUR}}$ recorded in human atrial myocytes. Thus, the block of the hKv1.5 channels by decursin would be an ideal antiarrhythmic drug specific for atrial fibrillation without the risk of Torsades de Pointes. The present study may provide some important idea for future development of antiarrhythmic drugs specific for atrial fibrillation.

\section{ACKNOWLEDGMENTS}

"This work was supported by the Grant of the Korean Ministry of Education, Science and Technology" (The Regional Core Research Program/Center for Healthcare Technology Development) and the Grant of Woosuk University.

\section{REFERENCES}

Choe, H., Lee, Y. K., Lee, Y. T., Choe, H., Ko, S. H., Joo, C. U., Kim M. H., Kim, G. S., Eun, J. S., Kim, J. H., Chae, S. W. and Kwak, Y. G. (2003) Papaverine blocks hKv1.5 channel current and human atrial ultrarapid delayed rectifier $\mathrm{K}^{+}$currents. J. Pharmacol. Exp. Ther. 304, 706-712.

Chung, S. S., Blackshear, J. L., Shen, W. K., Hammill, S. C. and Gersh, B. J. (2001) Epidemiology and natural history of atrial fibrillation: clinical implications. J. Am. Coll. Cardiol. 37, 371-378.

Eun, J. S., Choi, B. H., Park, J. A., Lee, G. I., Lee, T. Y., Kim, D. K., Jung, Y. H., Yoo, D. J. and Kwak, Y. G. (2005a) Open channel block of hKv1.5 by psoralen from Heracleum moellendorffii Hance. Arch. Pharm. Res. 28, 269-273.

Eun, J, S., Kim, K. S., Kim, H. N., Park, S. A., Ma, T. Z., Lee, K. A., Kim, D. K., Kim, H. K., Kim, I. S., Jung, Y. H., Zee, O. P., Yoo, D. J. and Kwak, Y. G. (2007) Synthesis of psoralen derivatives and their blocking effect of hKv1.5 channel. Arch. Pharm. Res. 30, 155-160.

Eun, J. S., Park, J. A., Choi, B. H., Cho, S. K., Kim, D. K. and Kwak, Y. G. (2005b) Effects of oxypeucedanin on hKv1.5 and action potential duration. Biol. Pharm. Bull. 28, 657-660.

Fedida, D., Chen, F. S. and Zhang, X. (1998) The 1997 Stevenson
Award Lecture. Cardiac $\mathrm{K}^{+}$channel gating: cloned delayed rectifier mechanisms and drug modulation. Can. J. Physiol. Pharmacol. 76, 77-89.

Jung, D. J., Porzel, A. and Huneck, S. (1991) Gigasol and other coumarines from Angelica gigas. Phytochemistry 30, 710-712.

Kang, S. Y., Lee, K. Y., Sung, S. H., Park, M. J. and Kim, Y. C. (2001) Coumarins isolated from Angelica gigas inhibit acetyl cholinesterase: Structure-activity relationships. J. Nat. Prod. 64, 683-685.

Kim, W. J., Lee, S. J., Choi, Y. D. and Moon, S. K. (2010) Decursin inhibits growth of human bladder and colon cancer cells via apoptosis, G1-phase cell cycle arrest and extracellular signal-regulated kinase activation. Int. J. Mol. Med. 25, 635-641.

Kwak, Y. G., Kim, D. K., Ma, T. Z., Park, S. A., Park, H., Jung, Y. H., Yoo, D. J. and Eun, J. S. (2006) Torilin from Torilis japonica (Houtt.) DC. blocks hKv1.5 channel current. Arch. Pharm. Res. 29, 834839.

Kwak, Y. G., Navarro-Polanco, R. A., Grobaski, T., Gallagher, D. J. and Tamkun, M. M. (1999) Phosphorylation is required for alteration of $\mathrm{Kv} 1.5 \mathrm{~K}+$ channel function by the Kvbeta 1.3 subunit. J. Biol. Chem. 274, 25355-25361.

Lee, H. J., Lee, H. J., Lee, E. O., Lee, J. H., Lee, K. S., Kim, K. H., Kim, S. H. and Lü, J. (2009) In vivo anti-cancer activity of Korean Angelica gigas and its major pyranocoumarin decursin. Am. J. Chin. Med. 37, 127-142.

Lee, S., Kang, S. S. and Shin, K. H. (2002) Coumarins and a pyrimidine from Angelica gigas roots. Nat. Prod. Sci. 8, 58-61.

Lee, S. H., Lee, Y. S., Jung, S. H., Shin, K. H., Kim, B. K. and Kang, S. S. (2003) Anti-tumor activities of decursinol angelate and decursin from Angelica gigas. Arch. Pharm. Res. 26. 727-730.

Pachaly, P., Treitner, A. and Sin, K. S. (1996) Neue cumaringlykoside aus Angelica gigas. Pharmazie 51, 57-61.

Perchenet, L. and Clement-Chomienne, O. (2000) Characterization of mibefradil block of the human heart delayed rectifier hKv1.5. J. Pharmacol. Exp. Ther. 295, 771-778.

Ryu, K. S., Hong, N. D., Kim, N. J. and Kong, Y. Y. (1990) Studies on the coumarin constituents of the root of Angelica gigas Nakai. Isolation of decursinol angelate and assay of decursin angelate and decursin. Korean J. Pharmcacogn. 21, 64-68.

Shin, K. H., Han, J. M. and Lee, I. R. (1996) Effect of the constituents of Angelica gigantis Radix on hepatic drug metabolizing enzymes. Korean J. Pharmcacogn. 27, 323-327.

Snyders, D. J., Tamkun, M. M. and Bennett, P. B. (1993) A rapidly activating and slowly inactivating potassium channel cloned from human heart. Functional analysis after stable mammalian cell culture expression. J. Gen. Physiol. 101, 513-543.

Wang, Z., Fermini, B. and Nattel, S. (1994) Rapid and slow components of delayed rectifier current in human atrial myocytes. Cardiovasc. Res. 28, 1540-1546.

Yook, C. S. (1990) Coloured medicinal plants of Korea. pp. 390. Academy Book, Seoul, Korea. 